\title{
Striking increase in the incidence of infective endocarditis associated with recreational drug abuse in urban South Africa
}

\author{
R Meel, PhD; M R Essop, MD \\ Division of Cardiology, Department of Internal Medicine, Chris Hani Baragwanath Academic Hospital and Faculty of Health Sciences, \\ University of the Witwatersrand and Milpark Hospital, Johannesburg, South Africa
}

Corresponding author: R Meel (ruchikameel@gmail.com)

\begin{abstract}
Background. We have recently noted a dramatic rise in the number of patients with infective endocarditis (IE) related to intravenous (IV) nyaope (a mixture of heroin, cocaine and antiretroviral drugs) presenting to Chris Hani Baragwanath Academic Hospital in Johannesburg, South Africa.

Objectives. To document the clinical and echocardiographic characteristics of these patients.

Methods. Clinical and echocardiographic characteristics of all patients $(N=68)$ with IE due to IV nyaope use were retrospectively extracted from hospital records (December 2014 - February 2017).

Results. The mean (standard deviation) age of the patients was 25.8 (4.5) years (97.1\% were male). Withdrawal symptoms were noted in $25.1 \%$ of cases, fever in $58.8 \%$, dyspnoea in $86.7 \%$ and right ventricular failure in $42.6 \%$. Most patients were HIV-positive (76.1\%), with CD4+ cell counts of $<200$ cells $/ \mu \mathrm{L}$ in $8.8 \%$ of the total, $58.1 \%$ had hepatitis $\mathrm{C}$ infection, and only three were on antiretrovirals. Septic pulmonary emboli were noted in $61.8 \%$. Blood cultures revealed Staphylococcus aureus in $61.2 \%$, Enterococcus faecalis in $8.8 \%$ and Pseudomonas aeruginosa in 1 patient; $29.2 \%$ had sterile cultures and $8.8 \%$ polymicrobial infection. Severe right ventricular systolic dysfunction (RVS Doppler velocity $<10 \mathrm{~cm} / \mathrm{s}$ ) and pulmonary hypertension were noted in $19.1 \%$ and $62.2 \%$ of patients, respectively. Pericardial effusion was noted in $37.8 \%$. The most commonly involved valve was the tricuspid (60.1\%), followed by the mitral (17.2\%), aortic (2.9\%) and pulmonary (1 patient) valves. Combined valve lesions were noted in $19.1 \%$ of patients. Ten patients (14.7\%) died. The main predictor of in-hospital mortality was $S$. aureus infection (odds ratio $5.0 ; p=0.042$ ).

Conclusions. We have documented the common clinical and echocardiographic characteristics of patients with IE secondary to IV nyaope use. IE due to IV drug use is responsible for considerable morbidity and mortality in a predominantly young male population.

S Afr Med J 2018;108(7):585-589. DOI:10.7196/SAMJ.2018.v108i7.13007
\end{abstract}

In developed countries, infective endocarditis (IE) frequently complicates intravenous drug use (IDU) and HIV infection. ${ }^{[1]}$ IE due to IDU has been rare in sub-Saharan Africa in both the pre- and post-HIV eras, probably owing to the low frequency of IDU. ${ }^{[1]}$ We have recently observed an increase in the number of patients with IE secondary to intravenous (IV) nyaope use presenting to Chris Hani Baragwanath Academic Hospital (CHBAH) in Johannesburg, South Africa (SA). ${ }^{[2]}$ Nyaope, also called whoonga (or wunga), is a drug sold on the streets and has been in widespread use in SA since about 2010, initially among poor communities in Durban. ${ }^{[3]}$ The ingredients are not exact or constant, but it usually contains an antiretroviral (ARV) such as efavirenz or ritonavir. ${ }^{[3]}$ Other components include heroin, crystal methamphetamine, cannabis, cocaine and even rat poison. ${ }^{\left[{ }^{[3]}\right.}$ Heroin is the primary substance of abuse for $8 \%$ of individuals in treatment centres in Gauteng Province, SA. Nyaope is the most common form in which heroin is used by black South Africans, mainly owing to its low cost. Users become addicted to the heroin component, but the neurological effects of efavirenz may play a role, as it is known to cause psychoactive symptoms. ${ }^{[3]}$ ARVs may also potentiate the hypnotic effects of the other ingredients. ${ }^{[3]}$ ARVs are sourced illegally from healthcare workers or are stolen from patients taking them. ${ }^{[3]}$ Misuse of ARVs in turn may cause the emergence of resistant HIV strains. ${ }^{[3]}$

Nyaope is exclusive to SA and its use is common among young and unemployed black people from poor socioeconomic backgrounds. ${ }^{[3,4]}$
Although nyaope was initially smoked, it is now being injected. This new pattern of use is likely to lead to an increase in cases of IE. ${ }^{[2]}$

\section{Objectives}

There is a paucity of data on IE secondary to IDU in Africa. Our objective was therefore to systematically document the clinical and echocardiographic profile of these patients.

\section{Methods}

This study of a retrospective case series of patients with IE secondary to IV nyaope use was conducted at $\mathrm{CHBAH}$, which serves the periurban community of Soweto and is a large tertiary referral centre for surrounding hospitals and clinics in Gauteng and adjacent provinces. Data on all patients who presented to the cardiology clinic with IE secondary to IV nyaope use between December 2014 and February 2017 were extracted from hospital records. We included all patients aged $\geq 18$ years with documented definite or possible IE based on the modified Dukes criteria and a history of IV nyaope use. IE cases not related to IV nyaope use were excluded from the study. Data were collected by manual review of patient files. The following information was collected: patient demographics, comorbidities, history of IV nyaope use and its duration, factors predisposing to IE, echocardiographic findings, IE-related complications and causative micro-organisms, as well as outcomes (death, surgery, medical cure) until hospital discharge. 
The study was approved by the University of the Witwatersrand Ethics Committee (ref. no. M160723).

\section{Statistical analysis}

Statistical analysis was performed with Statistica (version 12.5, series 0414 for Windows; Dell, USA). Continuous variables were expressed as means (standard deviations (SDs)) for normally distributed data or medians with interquartile ranges (IQRs) for non-parametric data. Categorical variables were expressed as percentages and Fisher's exact test was used to compare categorical variables. Multivariate analysis was performed using logistic regression with statistically or clinically significant variables from univariate analysis. The level of statistical significance was $p<0.05$. Additionally, clinically significant variables with a $p$-value $<0.2$ such as age $\geq 30$ years, pulmonary artery systolic pressure $\geq 35 \mathrm{mmHg}$, right ventricular systolic function (RVS') and infection by the most commonly encountered pathogen, Staphylococcus aureus, were included in the multivariate analysis.

\section{Results}

\section{Patient characteristics}

The main demographic and clinical characteristics are presented in Table 1. Most patients were HIV-positive (76.1\%) and not on ARV therapy (only three patients were on ARVs at presentation). Hepatitis $\mathrm{B}$ infection was present in $8.2 \%$ and hepatitis $C$ infection in $58.1 \%$. Two patients $(2.9 \%)$ required dialysis for renal failure.

The most important modes of clinical presentation are depicted in Fig. 1. In addition, nonspecific complaints such as headache, chest pain and cough were present in $2.1 \%, 31.2 \%$ and $57.4 \%$ of patients, respectively. The mean (SD) haemoglobin concentration was $9.4(2.2) \mathrm{g} / \mathrm{dL}$, the mean (SD) white cell count $14.4(7) \times 10^{9} / \mathrm{L}$ and the median platelet count $202.5 \times 10^{9} / \mathrm{L}$ (IQR 138.5 - 365.5). The median C-reactive protein level was elevated at $108.5 \mathrm{mg} / \mathrm{L}$ (IQR 54.5 - 205.0). The median CD4+ count was 437 cells/ $\mu \mathrm{L}$ (IQR 266 - 635). Only 8.8\% of patients had a CD $4+$ count $<200$ cells $/ \mu \mathrm{L}$. Systemic emboli (central nervous system and coronary artery embolisation) were noted in $11.8 \%(n=8)$ of the patients. Septic pulmonary emboli (Fig. 2) were noted in $61.8 \%(n=42)$. Additional pulmonary involvement occurred in the form of atelectasis, effusion, bullae, pneumothorax, alveolar infiltrates, cavitation and infarction. Prior antibiotic use before the diagnosis of IE was made was reported in $60.3 \%$ of the patients; $17.1 \%$ of these were

Table 1. Demographic and clinical characteristics of patients $(N=68)$ with infective endocarditis due to intravenous nyaope use

\begin{tabular}{ll}
\hline Age (years), mean (SD) & $25.8(4.5)$ \\
Male gender, \% & 97.1 \\
Level of education, mean & Grade 10 \\
Employed & None \\
Duration of drug use (months), median (IQR) & $48(24-72)$ \\
NYHA functional class (I/II - III/IV), \% & $12.6 / 80.2 / 7.3$ \\
Systolic blood pressure (mmHg), mean (SD) & $113.9(14.8)$ \\
Diastolic blood pressure (mmHg), mean (SD) & $68.7(11.2)$ \\
Pulse rate (bpm), mean (SD) & $104.3(22.0)$
\end{tabular}

$\mathrm{SD}=$ standard deviation; $\mathrm{IQR}=$ interquartile range; $\mathrm{NYHA}=$ New York Heart Association.

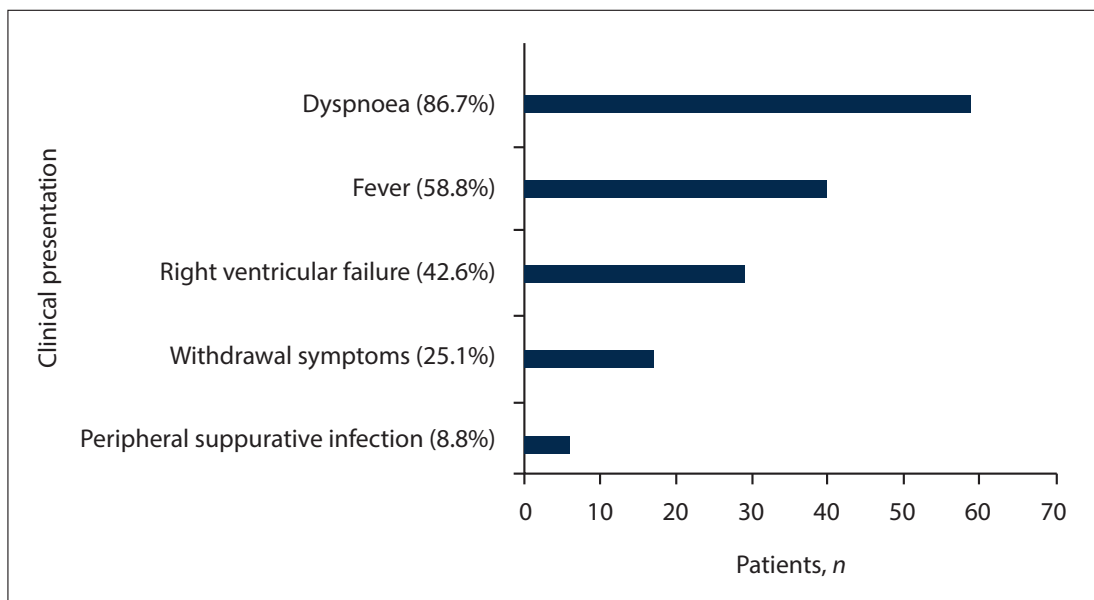

Fig. 1. Clinical presentation of patients with infective endocarditis due to intravenous nyaope use. erroneously treated for pneumonia before the diagnosis of IE. Pulmonary tuberculosis (PTB) was diagnosed in $36.3 \%$ of the patients, and two patients had coexisting systemic hypertension.

\section{Microbiological characteristics}

The most common micro-organisms cultured are shown in Fig. 3.

\section{Echocardiographic characteristics}

The median left ventricular ejection fraction (LVEF) was 64\% (IQR 55 - 68\%). Seven patients had an LVEF of $<50 \%$. The right ventricle (RV) was enlarged in $59.1 \%$ of the patients, with a mean (SD) RVS' Doppler velocity of $14.2(4.5) \mathrm{cm} / \mathrm{s}$. Severe RV systolic dysfunction was noted in 13 patients (RVS' Doppler velocity $<10 \mathrm{~cm} / \mathrm{s}$ ). The median pulmonary artery systolic pressure (PASP) was $35 \mathrm{mmHg}$ (IQR 23 - 42). Pulmonary hypertension (PHT) (PASP $\geq 35 \mathrm{mmHg}$ ) was present in $62.2 \%$ of the patients (Fig. 4). The mean (SD) vegetation size was 14.6 (7.7) $\mathrm{mm}$. In 20 patients the vegetation measured $<10 \mathrm{~mm}$. Twenty-five patients $(37.8 \%)$ had pericardial effusion. The most commonly involved valve was the tricuspid valve (TV) (60.1\%), followed by the mitral valve $(17.2 \%)$ and the aortic valve (AV) (2.9\%) (Fig. 5). The pulmonary valve was involved in only 1 patient. Combined valve lesions were noted in $19.1 \%$ of the patients. Severe tricuspid regurgitation (TR) was noted in 34 patients (50.0\%) (Fig. 4), moderate TR in 15 (22.1\%) and mild to trivial TR in the remaining 19 (27.9\%). Severe mitral regurgitation (MR) was noted in 8 patients $(11.7 \%)$ and moderate MR in 3 (4.4\%). Severe aortic regurgitation was present in 3 patients (4.4\%). Underlying rheumatic heart disease of the mitral valve was noted in 2 patients $(2.9 \%)$ and myxomatous disease was present in 1 . Less common sites included mural vegetations involving the right and left atrial walls in $3.0 \%$ of the patients, right ventricular outflow tract vegetations in 1 patient, isolated involvement of TV subvalvular apparatus in 1 , a vegetation on the aortomitral curtain in 1 and a periaortic root abscess as a complication in 2 .

\section{Follow-up and in-hospital mortality}

On in-hospital follow-up, it was discovered that 10 patients (14.7\%) had died and 4 (5.8\%) had absconded. Four patients underwent surgery; 1 underwent tricuspid valvulectomy, 1 mitral valve replacement, $1 \mathrm{TV}$ replacement and $1 \mathrm{AV}$ replacement. The remainder of the patients responded to 


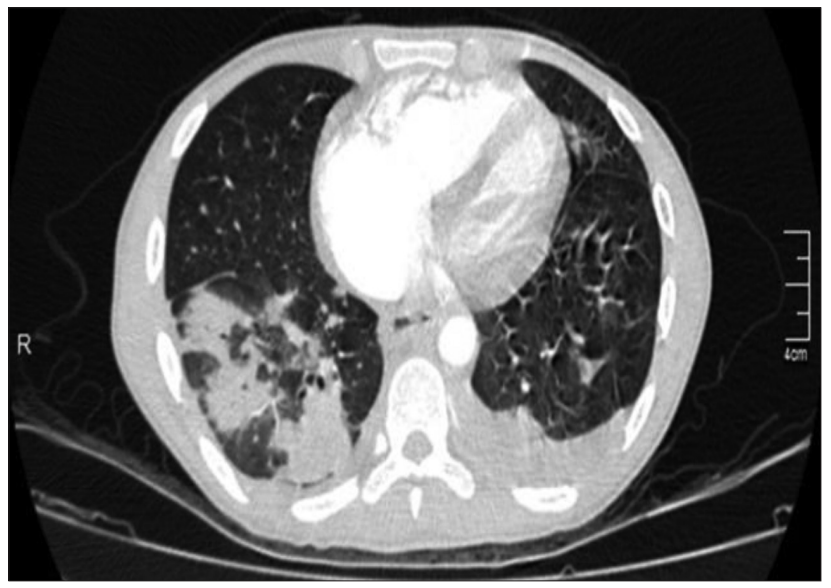

Fig. 2. Multiple areas of consolidation suggesting infarction due to septic pulmonary emboli and dilated right heart chambers on a computed tomography scan of the chest.

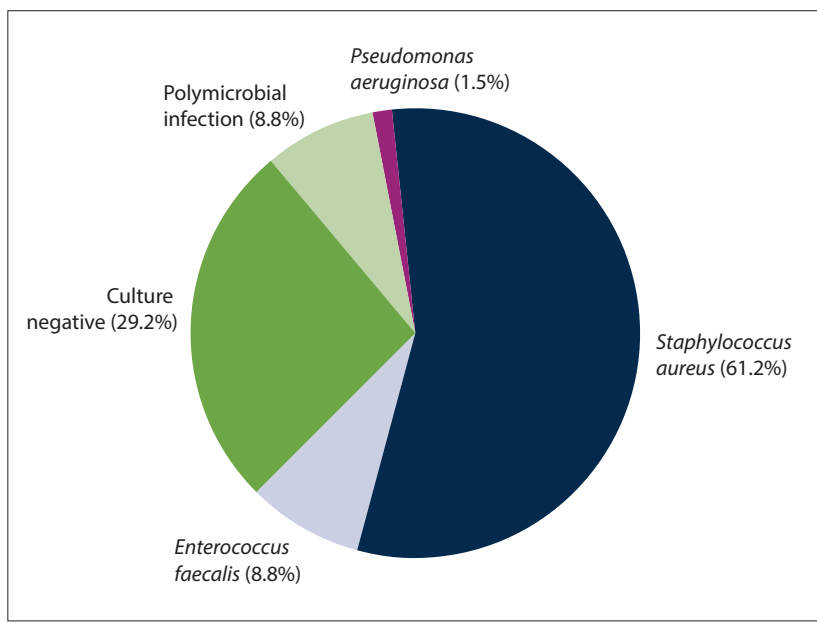

Fig. 3. Causative organisms in patients with infective endocarditis due to intravenous nyaope use.

antimicrobials and were culture-negative at discharge. Three patients presented with recurrent endocarditis. All the patients who died were HIV-positive. There was no statistically significant difference in in-hospital mortality between HIV-positive and HIV-negative patients $(p=0.06)$. The most important predictors that showed a trend in favour of increased in-hospital mortality on univariate analysis were $S$. aureus (odds ratio (OR) $3.5 ; p=0.06$ ) and Enterococcus faecalis infection (OR 4.6; $p=0.07$ ), and age $\geq 30$ years (OR 3.7; $p=0.05$ ). A CD4+ count $>200$ cells $/ \mu \mathrm{L}$ was a poor predictor of in-hospital mortality (OR $0.2 ; p=0.06$ ). Interestingly, variables such as PASP $\geq 35 \mathrm{mmHg}$ (OR $0.3 ; p=0.1$ ), vegetation size $\geq 10 \mathrm{~mm}$ (OR $0.5 ; p=0.4$ ) and severe TR (OR $1.5 ; p=0.5$ ) were not predictors of in-hospital mortality in this case series. On multivariate logistic regression analysis, S. aureus (OR 5.0; $p=0.04$ ) emerged as an important predictor of mortality when adjusted for PASP $\geq 35 \mathrm{mmHg}$, RVS' and age $\geq 30$ years (Table 2 ).

\section{Discussion}

This study represents the first case series reported on IE secondary to IV nyaope use in sub-Saharan Africa. The important findings of this study are as follows: (i) IE due to IV nyaope use is on the rise in a young, predominantly male population, most of whom present late because of delay on the part of the patient, late referral and misdiagnosis; (ii) hepatitis $\mathrm{C}$ co-infection and immunosuppression due to HIV were common findings in this group; (iii) methicillinsensitive $S$. aureus (MSSA) was the most common organism cultured and was associated with increased in-hospital mortality; E. faecalis, although less prevalent, showed a tendency towards increased in-hospital mortality; (iv) pulmonary hypertension, vegetation size and severe TR were poor predictors of in-hospital mortality; $(v)$ older age was associated with a trend towards increased mortality; and (vi) the surgical referral rate of patients with predominantly right-sided IE (RSIE) was very low.

We reported the first three cases of endocarditis associated with IV nyaope use in 2014. ${ }^{[2]}$ Since then we have noted a dramatic rise. Because of the social stigma associated with drug abuse and addiction to the drug, most of the patients in this case series presented late to the hospital. Lack of awareness among healthcare professionals about IE associated with IDU was one of the factors resulting in late referral from peripheral hospitals, as most of the patients in this study were erroneously treated for more commonly encountered conditions such as PTB and pneumonia, especially in the context of HIV. This resulted in problems such as partially treated endocarditis and recurrence, complications related to IE such as septic emboli, sterile blood cultures, delayed specialist referral, and ultimately suboptimal management. The reasons for delayed diagnosis include application of Dukes criteria for the diagnosis of RSIE, which may not be applicable, as the sensitivity and specificity of the modified Dukes criteria in RSIE has not been studied. ${ }^{[5]}$ In the context of RSIE, inclusion of septic pulmonary infarcts as a minor criterion in the modified Dukes criteria may therefore be inappropriate and these should rather form part of the major criteria.

Consistent with other recent studies, hepatitis $\mathrm{C}$ co-infection was common in this group of patients. ${ }^{[6,7]}$ Hepatitis $\mathrm{C}$ co-infection poses numerous problems; in addition to long-term risks such as liver cirrhosis and cancer, and transmission of infection to others, it also presents ethical dilemmas with regard to surgery in IDUs. ${ }^{[8,9]}$ In addition, significant costs are associated with the treatment of hepatitis C infection, often in non-compliant patients, who frequently have other concurrent opportunistic infections associated with HIV. ${ }^{[10]}$

In agreement with previous literature on IE in IDUs, the majority of the patients in this study were HIV-positive. ${ }^{[1]}$ The difference between the mortality rate in our HIV-positive patients and those who were HIV-negative was not statistically significant. This finding is consistent with the study on IV drug users by Ribera et al. ${ }^{[1]}$ In addition, in an SA study no difference in clinical and echocardiographic characteristics was noted between HIV-positive and HIV-negative patients with IE; however, none of them was noted to have a history of IDU. ${ }^{[12]}$ The lower mortality in HIV-positive compared with HIV-negative patients in the current study may partly be due to a smaller HIV-negative sample, and also to the fact that the majority had a CD $4+$ count $>200$ cells $/ \mu \mathrm{L}$. Furthermore, as expected in HIV-infected patients, a higher CD4+ count was associated with a lower in-hospital mortality rate, in agreement with findings from previous studies. ${ }^{[5]}$

Similar to other studies in HIV-infected IV drug users, S. aureus was the most common pathogen in this study. ${ }^{[13]}$ In contrast to the developed world, where methicillin-resistant $S$. aureus is an important causal pathogen of IE, MSSA is still the most prevalent organism associated with IE in IDUs in our setting. This is probably due to the early stage of the occurrence of this disease in SA, highly resistant organisms due to injudicious use of antibiotics not yet having emerged. ${ }^{[14]}$ Mortality rates of $8-12 \%$ associated with $S$. aureus IE in IDUs are reported in the literature. ${ }^{[15]}$ In the current case series 
with predominantly RSIE, mortality was higher than generally reported. In contrast to the study by Asgeirsson et al., ${ }^{[15]}$ we noted increased in-hospital mortality due to $S$. aureus associated with IDU. ${ }^{[15]}$ They reported an in-hospital mortality rate of $2.5 \%$, compared with $14.7 \%$ in our case series. The reason for our higher in-hospital mortality was probably delayed presentation and suboptimal care of these patients prior to referral to a tertiary care centre.

We found Enterococcus IE to be less common than MSSA, but a trend towards increased in-hospital mortality was associated with this organism. The association with high mortality due to Enterococcus endocarditis has been noted in previous studies. ${ }^{[16-18]}$ Because of the aggressive nature of IE associated with Enterococcus and $S$. aureus in our setting, initial empirical antibiotic therapy should cover both these organisms.

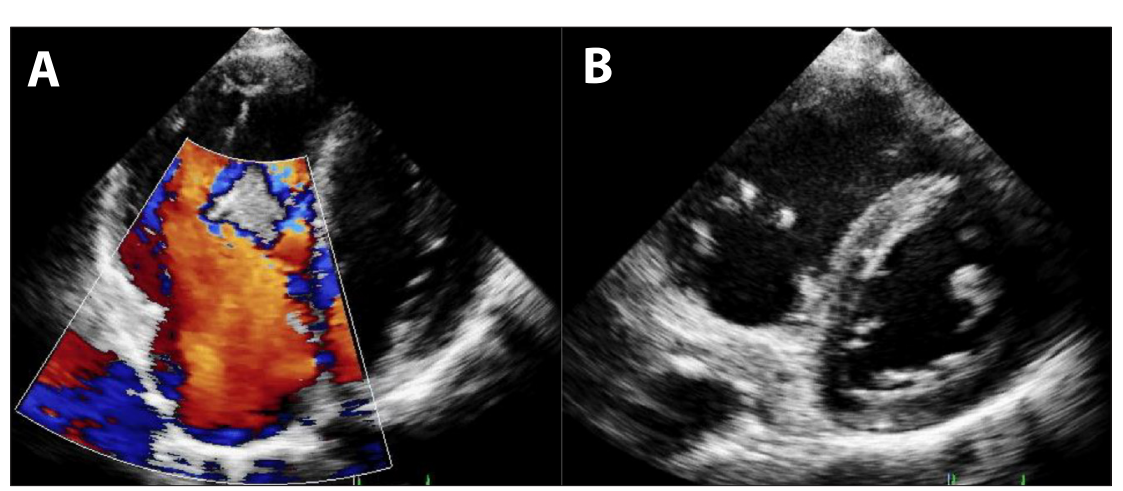

Fig. 4. (A) Severe pulmonary hypertension (with flattened interventricular septum) due to septic pulmonary emboli. (B) Severe tricuspid regurgitation secondary to tricuspid valve destruction and pulmonary hypertension.

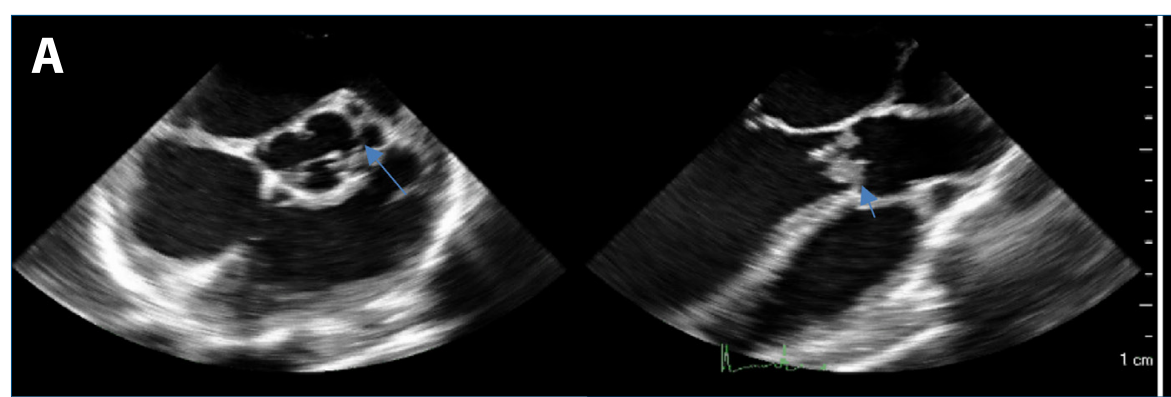

is probably because a significant portion of the vegetation had probably embolised to the lungs in patients with small vegetations on presentation, compared with those with larger vegetations.

We found that older age was associated with a trend towards increased in-hospital mortality. This was probably due to longer duration of drug use by individuals $>30$ years of age and therefore more complications (such as greater lung destruction due to chronic embolisation and comorbidities) on hospital admission.

Referral for surgery was low in our series, as the majority of patients responded to antimicrobial therapy or refused surgery. TV valvulectomy, nowadays generally considered of historical interest only, was performed in 1 case, as this patient had a long history of drug abuse and a high probability of recurrent endocarditis due to resumption of IDU. He was discharged and was lost to follow-up. Another 3 patients underwent valve replacement and remained well on follow-up. None of these 4 patients underwent valve repair, as the valves were significantly destroyed. Whether surgery should be performed in IDUs has been a subject of debate, and it is our opinion that this decision must be individualised, especially in a resource-limited setting. According to the limited available literature, surgery should be offered for the first episode of IE in IDUs who are willing to undergo rehabilitation. If the patient presents with a second episode of IE secondary to lack of compliance, he or she should probably not qualify for further surgical intervention. ${ }^{[2]]}$

Fig. 5. Echocardiographic images showing (A) multiple vegetations on the aortic valve complicated by a root abscess (arrows), and (B) a large vegetation on the tricuspid valve with extensive valve destruction (arrow).

Table 2. Possible predictors for mortality among hospitalised intravenous nyaope users

\begin{tabular}{|c|c|c|c|c|}
\hline \multirow[b]{2}{*}{ Variables } & \multicolumn{2}{|c|}{ Univariate } & \multicolumn{2}{|c|}{ Multivariate } \\
\hline & OR $(95 \% \mathrm{CI})$ & $p$-value & OR $(95 \% \mathrm{CI})$ & $p$-value \\
\hline Age $\geq 30$ years & $3.7(0.95-14.7)$ & 0.06 & $4.13(0.89-19.17)$ & 0.07 \\
\hline Staphylococcus aureus & $3.5(0.07-1.1)$ & 0.06 & $5.07(1.06-24.2)$ & 0.04 \\
\hline PASP $\geq 35 \mathrm{mmHg}$ & $0.34(0.09-1.2)$ & 0.11 & $0.25(0.05-1.19)$ & 0.08 \\
\hline RVS' tissue Doppler velocity $(\mathrm{cm} / \mathrm{s})$ & $0.5(0.09-3.17)$ & 0.17 & $0.39(0.05-2.81)$ & 0.35 \\
\hline
\end{tabular}




\section{Study limitations}

The main limitations of this study are: $(i)$ its retrospective design; (ii) its single-centre nature, which means that the results may not be generalisable to the larger population; and (iii) a small sample size and suboptimal follow-up owing to patient non-compliance.

\section{Conclusions}

We have documented the common clinical and echocardiographic characteristics of patients with IE secondary to IV nyaope use. IE due to IDU is a cause of considerable morbidity and mortality in a predominantly young male population. A high degree of suspicion must be maintained in order to make an early diagnosis and initiate treatment before embolic complications and heart failure intervene. Possible solutions include preventive education campaigns targeting individuals at risk of drug abuse. Early identification of addicted individuals and implementation of rehabilitation, cognitivebehavioural therapy and pharmacological treatment are critical. Increasing awareness among healthcare workers about IE due to IV drug abuse through education campaigns, emphasising early diagnosis and treatment of IE, is also important. Lastly, establishing a multidisciplinary endocarditis team would facilitate optimal patient management and outcomes. ${ }^{[20]}$

Acknowledgements. None.

Author contributions. RM collected data and conceptualised and wrote the manuscript. MRE conceptualised, co-authored and reviewed the manuscript. Funding. None.

Conflicts of interest. None.

1. Akinosoglou K, Apotolakis E, Marangos M, et al. Native right sided infective endocarditis. Eur J Intern Med 2013;24(6):510-519. https://doi.org/10.1016/j.ejim.2013.01.010

2. Meel R, Peters F, Essop MR. Tricuspid valve endocarditis associated with intravenous nyaope use: A report of 3 cases. S Afr Med J 2014;104(12):853-855. https://doi.org/10.7196/SAMJ.8291

3. Whoonga. Wikipedia encyclopedia. https://en.wikipedia.org/wiki/Whoonga (accessed 20 September 2017).
4. Rough K, Dietrich J, Essien T, et al. Whoonga and the abuse and diversion of antiretrovirals in Soweto, South Africa. AIDS Behav 2014;18(7):1378-1380. https://doi.org/10.1007/s10461-013-0683-x

5. Habib G, Hoen B, Tornos P, et al. Guidelines on prevention, diagnosis and treatment of infective endocarditis. Eur Heart J 2009;30(19):2369-2413. https://doi.org/10.1093/eurheartj/ehp285

6. Dawood MY, Cheema FH, Ghoreishi M, et al. Contemporary outcomes of operations for tricuspid . Dawood MY, Cheema FH, Ghoreishi M, et al. Contemporary outcomes of operations for tricuspid
valve infective endocarditis. Ann Thorac Surg 2015;99(2):539-546. https://doi.org/10.1016/j. valve infective endocaris
athoracsur.2014.08.069

7. Devi KhS, Brajachand N, Singh HL, et al. Co-infection by human immunodeficiency virus, hepatitis B and hepatitis C virus in injecting drug users. J Commun Dis 2005;37(1):73-77.

8. Sulkowski MS, Thomas DL. Epidemiology and natural history of hepatitis C virus infection in injection drug users: Implications for treatment. Clin Infect Dis 2005;40(Suppl 5):S263-S269. https:// doi.org/10.1086/427440

9. Gansera LS, Eszlari E, Deutsch O, et al. High-risk cardiac surgery in patients with intravenous drug abuse and/or active hepatitis $\mathrm{C}$ or HIV infection: An ethical discussion of six cases. Thorac Cardiovasc Surg 2016;64(1):2-5. https://doi.org/10.1055/s-0035-1551682

10. Werb D, Wood E, Kerr T, et al. Treatment costs of hepatitis $\mathrm{C}$ infection among injection drug users in Canada, 2006 - 2026. Int J Drug Policy 2011:22(1):70-76. https://doi.org/10.1016/j.drugpo 2010.09.006

1. Ribera $\mathrm{E}$ Miró $\mathrm{JM}$ Cortés $\mathrm{E}$ et al. Influence of human inmunodeficiency virus 1 infection and deree of im degree of immunosuppression in the clinical characteristics and outcome of infective endocarditis
in intravenous drug users. Arch Intern Med 1998;158(18):2043-2050. https://doi.org/10.1001/ in intravenous drug
archinte. 158.18 .2043

12. Nel SH, Naidoo DP. An echocardiographic study of infective endocarditis, with special reference to patients with HIV. Cardiovasc J Afr 2014;25(2):50-57. https://doi.org/10.5830/CVJA-2013-084

13. Valencia E, Miro J. Endocarditis in the setting of HIV infection. AIDS Rev 2004;6(2):97-106.

4. Tong SYC, Davis JS, Eichenberger E, et al. Staphylococcus aureus infections: Epidemiology, pathophysiology, clinical manifestations, and management. Clin Microbiol Rev 2015;28(3):603-661. https://doi.org/10.1128/CMR.00134-14

15. Asgeirsson $\mathrm{H}$, Thalme A, Weiland O. Low mortality but increasing incidence of Staphylococcus aureus endocarditis in people who inject drugs: Experience from a Swedish referral hospital. Medicine (Baltimore) 2016;95(49):e5617. https://doi.org/10.1097/MD.0000000000005617

16. Demin AA, Drobysheva VP, Vel'ter O. Infectious endocarditis in intravenous drug abusers. Klin Med (Mosk) 2000;78(8):47-51.

17. Fernández Guerrero ML, Goyenechea A, Verdejo C, et al. Enterococcal endocarditis on native and prosthetic valves: A review of clinical and prognostic factors with emphasis on hospital-acquired infections as a major determinant of outcome. Medicine (Baltimore) 2007;86(6):363-377. https://doi. infections as a major determinant of
org/10.1097/MD.0b013e31815d5386

18. Martínez-Marcos FJ, Lomas-Cabezas JM, Hidalgo-Tenorio C, et al. Enterococcal endocarditis Aartínez-Marcos FJ, Lomas-Cabezas JM, Hidalgo-Tenorio C, et al. Enterococcal endocarditis:
A multicentre study of 76 cases. Enferm Infecc Microbiol Clin 2009;27(10):571-579. https://doi. A multicentre study of 76 cases. Enferm Infecc Microbiol Clin 2009;27(10):571-579. https://doi. org/10.1016/j.eimc.2009.02.007

19. Mi M, Nelson S, Weiner R. Clinical and echocardiographic factors associated with in-hospital mortality in patients with infective endocarditis affecting the native tricuspid valve. Am J Cardio 2016;118(5):739-743. https://doi.org/10.1016/j.amjcard.2016.06.011

20. Habib G, Lancellotti P, Antunes MJ, et al., Task Force for the Management of Infective Endocarditi of the European Society of Cardiology. 2015 ESC Guidelines for the management of infective endocarditis. G Ital Cardiol 2016;17(4):277-319. https://doi.org/10.1714/2214.23904

21. Yeo KK, Chang WJ, Lau JM, et al. Valve replacement in endocarditis: Setting limits in noncompliant intravenous drug abusers. Hawaii Med J 2006;65(6):168,170-171. 